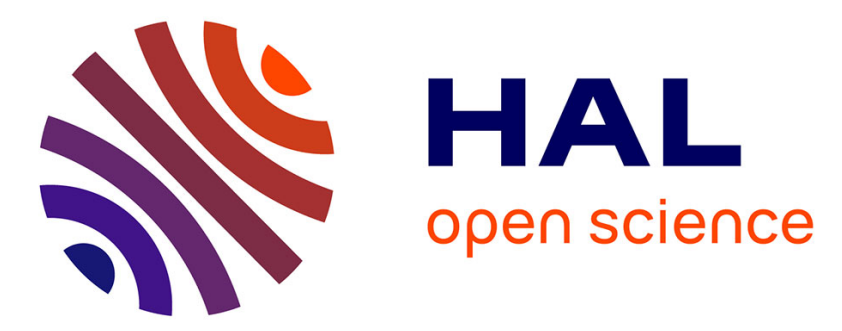

\title{
All-optical technique to measure the pyroelectric coefficient in electro-optic crystals
}

J. Parravicini, J. Safioui, V. Degiorgio, P. Minzioni, M. Chauvet

\section{To cite this version:}

J. Parravicini, J. Safioui, V. Degiorgio, P. Minzioni, M. Chauvet. All-optical technique to measure the pyroelectric coefficient in electro-optic crystals. British Journal of Applied Physics, 2011, 109 (3), pp.033106. 10.1063/1.3544069 . hal-00577206

\section{HAL Id: hal-00577206 \\ https://hal.science/hal-00577206}

Submitted on 12 Apr 2021

HAL is a multi-disciplinary open access archive for the deposit and dissemination of scientific research documents, whether they are published or not. The documents may come from teaching and research institutions in France or abroad, or from public or private research centers.
L'archive ouverte pluridisciplinaire HAL, est destinée au dépôt et à la diffusion de documents scientifiques de niveau recherche, publiés ou non, émanant des établissements d'enseignement et de recherche français ou étrangers, des laboratoires publics ou privés. 


\title{
All-optical technique to measure the pyroelectric coefficient in electro-optic crystals
}

\author{
Jacopo Parravicini, ${ }^{1,2, a)}$ Jassem Safioui, ${ }^{1}$ Vittorio Degiorgio, ${ }^{3}$ Paolo Minzioni, ${ }^{3}$ and \\ Mathieu Chauvet ${ }^{1}$ \\ ${ }^{1}$ Institut FEMTO-ST-Département d'Optique "P.M. Duffieux," Université de Franche-Comté, \\ 16 Route De Gray, 25030 Besançon, France \\ ${ }^{2}$ Dipartimento di Ingegneria Elettrica e dell'Informazione, Università degli Studi de L'Aquila - Via \\ Gronchi 18, 67100 L'Aquila, Italy \\ ${ }^{3}$ CNISM and Dipartimento di Elettronica, Università degli Studi di Pavia, Via Ferrata 1, 27100 Pavia, Italy
}

We report an all-optical method to measure the pyroelectric coefficient $p$ of electro-optic crystals. Through this technique, we first acquire the birefringence variation $\delta \Delta n$ of the crystal as a function of its temperature $T$, both in closed and open-circuit conditions, using a Sénarmont phase-compensation configuration. Then the pyroelectric field is deduced from the difference between these two measurements, so it leads to the material spontaneous polarization change, whose derivative with respect to the temperature finally gives $p$. This technique is applied on congruent and stoichiometric lithium niobate.

\section{INTRODUCTION}

A ferroelectric crystal exhibiting variation in spontaneous polarization $P_{s}$ as a function of the temperature $T$ is called pyroelectric. Such a change in polarization is expressed by the pyroelectric coefficient $p$, which is defined through ${ }^{1}$ the relationship,

$$
p=\frac{\partial P_{s}}{\partial T} .
$$

In such crystals, the relation between the electric displacement $D$ and the electric field $E$ can be expressed through the equation $D=P_{s}+\varepsilon E$ (where $\varepsilon$ is the dielectric constant). In an open-circuit configuration, a polarization change due to a temperature change can arise with no change in dielectric displacement. In such a situation, we can write

$$
0=\Delta D=\Delta P_{s}+\varepsilon \Delta E .
$$

As a consequence an electric field $E_{p y}$ arises in the crystal, being

$$
E_{p y}=\Delta E=-\frac{1}{\varepsilon} \Delta P_{s},
$$

which can be expressed by introducing the pyroelectric coefficient in Eq. (1) to give

$$
E_{p y}=-\frac{p}{\varepsilon} \Delta T .
$$

In fact, e.g., in lithium niobate, one of the most widely studied ferroelectric material, ${ }^{2}$ such a field was recently used to generate bright solitons, so called pyrolitons. ${ }^{3,4}$

Actually, pyroelectricity being a dielectric phenomenon, the pyroelectric coefficient is usually obtained through dielectric experiments, which generally employ a configuration

\footnotetext{
${ }^{a)}$ Electronic mail: jacopo.parravicini@unipv.it.
}

where two electrodes are placed on the opposite polar faces of the sample. ${ }^{1,5-8}$ The coefficient is then obtained by measuring either the charge or the current established as a consequence of the pyroelectric field $E_{p y}$ (i.e., of the potential difference) generated by the temperature variation. ${ }^{1,9}$ However, dielectric measurements are well known to present different experimental difficulties. ${ }^{9}$ Namely, in the case of lithium niobate, e.g., they give a spreading of the measured values of the pyroelectric coefficient $p .^{10}$ The present work is, therefore, motivated by the quest of a different (not dielectric) technique which can give peculiar information on the pyroelectric properties of a crystal. Specifically, we present a new all-optical technique to determine the pyroelectric coefficient based on measurement of birefringence variation; the procedure we devised can be used in pyroelectric crystals that also present electro-optic (Pockels) effect and it is an alternative to the usual (quasi) static dielectric measurements.

\section{PRINCIPLES OF THE TECHNIQUE}

Let us consider a pyroelectric crystal affected by the Pockels effect. If it suffers a temperature variation $\Delta T$, it will present a variation $\delta \Delta n$ of birefringence, which can be written as the sum of two contributions, $\delta \Delta n_{t o}$ and $\delta \Delta n_{p y}$. The first one is the birefringence variation due to the thermooptic effect, i.e., the usual change in refractive indices due to their dependence on temperature, while the second one is the contribution generated by the pyroelectric field $E_{p y}$ through the Pockels effect. So we can write

$$
\delta \Delta n=\delta \Delta n_{t o}+\delta \Delta n_{p y} .
$$

In open-circuit conditions the pyroelectric field arises simultaneously with the temperature variation. Once the steadystate temperature is reached, it is slowly compensated by charges sorption on the polar surfaces of the crystal. 


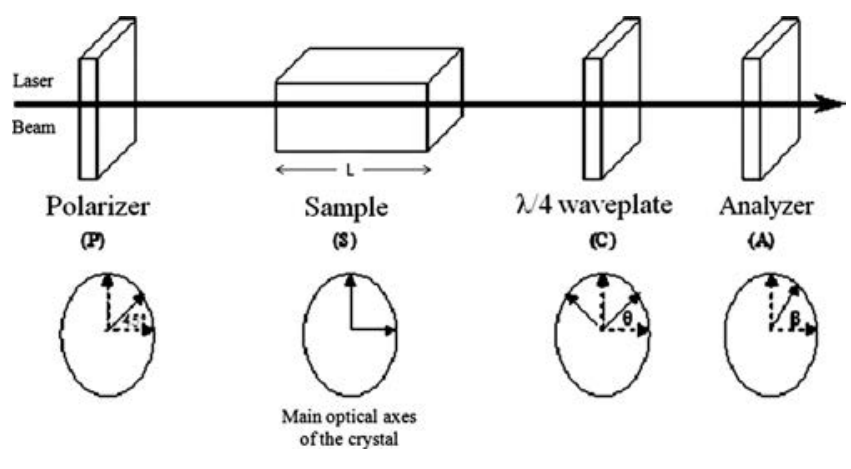

FIG. 1. Sénarmont configuration with its components orientation.

The first step of our method is to measure the total birefringence variation $\delta \Delta n$ induced by a temperature change $\Delta T$ before any compensation of $E_{p y}$ takes place. In a second step the thermo-optic term $\delta \Delta n_{t o}$ is evaluated. It can be done either experimentally, by short-circuiting the polar facets of the crystal, which establishes a null field, or analytically by using Sellmeier equations. Subtraction of the thermo-optic contribution from the total birefringence finally provides the pyroelectric contribution $\delta \Delta n_{p y}$ [Eq. (5)]. For electro-optic materials ${ }^{11}$ the relationship holds

$$
\delta \Delta n_{p y} \propto E_{p y} .
$$

If electro-optic coefficients and refractive indices are known, it will be straightforward ${ }^{11}$ to deduce the pyroelectric field $E_{p y}$. Finally the pyroelectric coefficient $p$ is calculated from Eq. (1).

In this paper, the Sénarmont technique is used to measure the birefringence variation as a function of temperature. Note that an interferometric setup could also be used to detect the refractive indices change. We apply our all-optical method to determine $\mathrm{LiNbO}_{3}$ pyroelectric properties. Before presenting the procedure and the results on this material, we recall the basis of Sénarmont method.

\section{THE SÉNARMONT METHOD}

The Sénarmont phase-compensation method, schematically depicted in Fig. 1 and described in Refs. 11 and 12, employs a low-power (tens of $\mu \mathrm{W}$ ) laser beam of about $80 \mu \mathrm{m}$ in diameter as a probe. The crystal to be analyzed (S) is placed between two linear polarizers, the first one $(\mathrm{P})$ is set to yield a beam polarization at $45^{\circ}$ with respect to the optical axis $\hat{c}$ of the crystal, while the second one (A) is the analyzer. A $\lambda / 4$ waveplate $(\mathrm{C})$ is placed between the sample and the analyzer, with its optical axis parallel to that of the polarizer (P). The probe-beam passes through the sample whose birefringence is to analyze. At the exit, the phase shift $\Gamma$ between its ordinary and extraordinary polarization components is proportional to the birefringence $\Delta n:{ }^{11-13}$

$$
\Gamma=\frac{2 \pi}{\lambda_{p}} L \Delta n,
$$

where $\lambda_{p}$ is the wavelength of the laser beam and $L$ is the length of the sample. If $\beta$ is the angle of the analyzer (A) with respect to $\hat{c}$ axis, in the configuration described above, the transmission function $\tau(\Gamma, \beta)$ is given by ${ }^{12}$

$$
\tau=\frac{1}{2}[1 \pm \sin (\Gamma \pm 2 \beta)],
$$

where the relationship $|d \beta / d \Gamma|=2$ holds. The former equation provides the following relationship between a variation in phase-shift $\Delta \Gamma$ and a measured variation in angle analyzer $\Delta \beta$

$$
\Delta \Gamma=\frac{1}{2} \Delta \beta .
$$

Equations (7) and (9) can be combined to deduce any birefringence variation $\delta \Delta n$ from a measurement of $\Delta \beta$

$$
\delta \Delta n=\frac{\lambda_{p}}{\pi L} \Delta \beta .
$$

\section{EXPERIMENTS ON LITHIUM NIOBATE: PROCEDURE}

In lithium niobate the pyroelectric field, $E_{p y}$, arises in direction of the ferroelectric axis, ${ }^{4}$ which is parallel to the optical axis of the crystal, then it produces a birefringence variation $\delta \Delta n_{p y},{ }^{11}$

$$
\delta \Delta n_{p y}=-\frac{1}{2}\left(n_{e}^{3} r_{33}-n_{o}^{3} r_{13}\right) E_{p y},
$$

where $n_{e}$ and $n_{o}$ are, respectively, the extraordinary and the ordinary refractive indices and $r_{33}$ and $r_{13}$ are the suitable crystal electro-optic coefficients.

The experimental procedure is detailed below.

(1) The crystal is placed in a configuration as in Fig. 1; the beam propagation direction is parallel to one of ordinary optical axis and perpendicular to the $\hat{c}$-axis while its polarization direction is inclined of $45^{\circ}$ with respect to the $\hat{c}$-axis. The sample is attached to a holder that can heat at a controlled rate.

(2) During the heating time, through the Sénarmont setup the system follows the evolution of maxima, minima and inflexions of $\tau$ [Eq. (8)], and consequently the evolution of $\beta$ as a function of the temperature $T$.

(3) The obtained variation in angle analyzer $\Delta \beta$ is recorded as a function of $T$. It provides the phase-shift variation $\Delta \Gamma$ and then the birefringence variation in the sample $\delta \Delta n=\delta \Delta n(T)$ is straightforward calculated by the Eq. (10).

(4) In order to deduce the pyroelectric contribution $\delta \Delta n_{p y}$ from $\delta \Delta n$, it is necessary to estimate and subtract the thermo-optic contribution $\delta \Delta n_{t o}$. It is possible to estimate theoretically $\delta \Delta n_{t o}(T)$ by using the Sellmeier equations. On the other hand, to obtain $\delta \Delta n_{t o}$ experimentally, e.g., when crystals with unknown Sellmeier equations are considered, the birefringence variation can be measured with the Sénarmont setup but using a shortcircuited sample in order to get a null pyroelectric field $E_{p y}$. Consequently measurement of the sample birefringence variation provides the sole thermo-optic contribution $\delta \Delta n_{t o}=\delta \Delta n_{t o}(T)$. In our experiments in lithium niobate, reported below, we have tested both procedures, which have given very similar results. 
(5) From $\delta \Delta n_{p y}(T)$ the pyroelectric field $E_{p y}(T)$ can be deduced using Eq. (11),

$E_{p y}(T)=-2 \frac{\delta \Delta n_{p y}(T)}{\left[n_{e}^{3}(T) r_{33}(T)-n_{o}^{3}(T) r_{13}(T)\right]}$.

It is worth noting that to obtain accurate values of $E_{p y}$ by application of the relationship (12), it is necessary to precisely know the refractive indices and electro-optic coefficients varying as a function of temperature.

(6) From $E_{p y}(T)$, the spontaneous polarization variation $\Delta P_{s}(T)$ generated by the pyroelectric effect can be calculated using Eq. (3) knowing the specific dielectric functions, taken from the literature.

(7) Finally the pyroelectric coefficient $p$ of the considered material is obtained from $P_{s}(T)$ by calculating its derivative with respect to the temperature, following Eq. (1).

The procedure leads to the net value of the pyroelectric field for any established temperature variation, providing negligible compensation of pyroelectric field by charge sorption. We stress that such an experimental technique produces almost no perturbation to the system since a low-power beam is used. The pyroelectric field is, therefore, not influenced by the measurement process because the measured system (an electric field generated by charges) is not perturbed by the measuring system (a light beam).

\section{EXPERIMENTS ON LITHIUM NIOBATE: RESULTS AND DISCUSSION}

To demonstrate the technique, we apply it to study lithium niobate. Actually, in the literature, as we hinted, there is a large discrepancy among different values of its pyroelectric coefficient $p .^{10}$

Our Sénarmont apparatus (Fig. 1), described in Ref. 14, employs a He-Ne laser source and as analyzer (A) a rotating polarizer, whose angular position is computer-controlled, while the transmitted light is detected with a photodiode. Measurement sensitivity is enhanced using a lock-in detection technique. This apparatus allows following continuously the polarization direction of the exiting beam as a function of time, acquiring nearly 450 readings of the polarization angle each minute. We have investigated commercial $\mathrm{LiNbO}_{3}$ crystals in both stoichiometric and congruent compositions with a propagation length of $5 \mathrm{~mm}$ and $2.8 \mathrm{~mm}$, respectively.

To apply the previously described experimental procedure, we first place the sample on a metallic base equipped with a programmable electric heater and covered by a metallic lid with small apertures for beam laser access. To improve sample thermalization, its face in contact with the heating plate is coated with silver paint. Temperature evolution is monitored through a thermocouple placed below the sample. Temperature is gradually increased from 27 to $47^{\circ} \mathrm{C}$, at the rate of $0.44{ }^{\circ} \mathrm{C} / \mathrm{min}$. To avoid any problem for recording system stability or charge sorption, we use a $20^{\circ} \mathrm{C}$ interval, which can be covered in a relatively short time (nearly 45 min) employing a slow heating rate (necessary to get a good

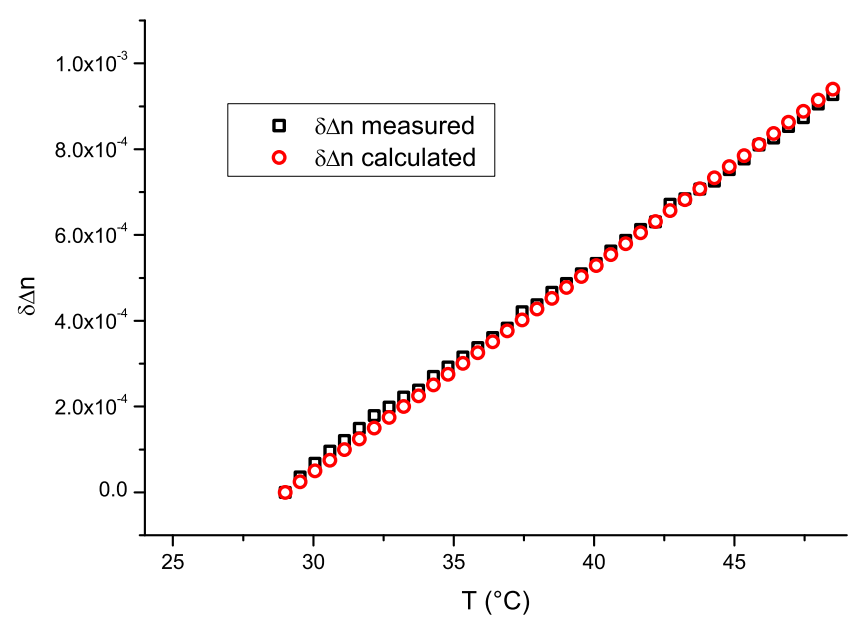

FIG. 2. (Color online) Typical birefringence variation $\delta \Delta n$ measurement in a stoichiometric crystal in short-circuit configuration (black squares) in comparison with calculated $\delta \Delta n$ (red circles).

thermalization). During the heating process, we continuously record the angle of polarization $\beta$, and we deduce immediately $\delta \Delta n$ through Eq. (10) from $\Delta \beta$.

A first set of experiments, on both stoichiometric and congruent crystals, is carried out in short-circuit conditions, in order to cancel $E_{p y}$ and obtain the pure thermo-optic contribution. Such conditions, where $\delta \Delta n=\delta \Delta n_{t o}$ holds, are achieved by coating with silver paint both lower and upper $\hat{c}$-faces of the sample and connecting them. A typical birefringence variation measurement $\delta \Delta n$ as a function of temperature is reported in Fig. 2. In the same figure the birefringence variation obtained from the Sellmeier equations ${ }^{15}$ is depicted. We observe a very good agreement between measured and calculated values: such a result validates our hypothesis, the discrepancy between measured and predicted data being less than $5 \%$.

Therefore, since Sellmeier equations provide accurately the birefringence variation due to thermo-optic effect $\delta \Delta n_{t o}$, this contribution will be calculated instead of measured when needed in the following part of this work.

A second set of experiments is carried out in a crystal in open-circuit configuration, to estimate $E_{p y}$. In Fig. 3 typical results obtained in a stoichiometric $\mathrm{LiNbO}_{3}$ sample are reported. The graph presents the measured total birefringence $\delta \Delta n$ variation together with the calculated thermo-optic contribution $\delta \Delta n_{t o}$, and the pyroelectric contribution $\delta \Delta n_{p y}$ obtained by subtracting the two latter curves. We observe that the birefringence variation due to the pyroelectric effect is opposite in sign to the thermo-optic contribution. The negative value of $\delta \Delta_{p y}$ is consistent with a decrease in the material spontaneous polarization $P_{s}$ when the temperature increases, as expected in pyroelectric crystals. The magnitude of $\delta \Delta n_{t o}$ is observed to be about twice the amplitude of $\delta \Delta n_{p y}$. From $\delta \Delta n_{p y}$ we calculate the pyroelectric field $E_{p y}$ using the relationship (12). To this end, the refractive indices $n_{e}$ and $n_{o}$ dependence versus temperature is given by Sellmeier equations. ${ }^{15}$ The variation in the electro-optic coefficients in the temperature interval is considered negligible in accordance with recent publication. ${ }^{16}$ Then the material spontaneous polarization change $\Delta P_{s}$ can be calculated 


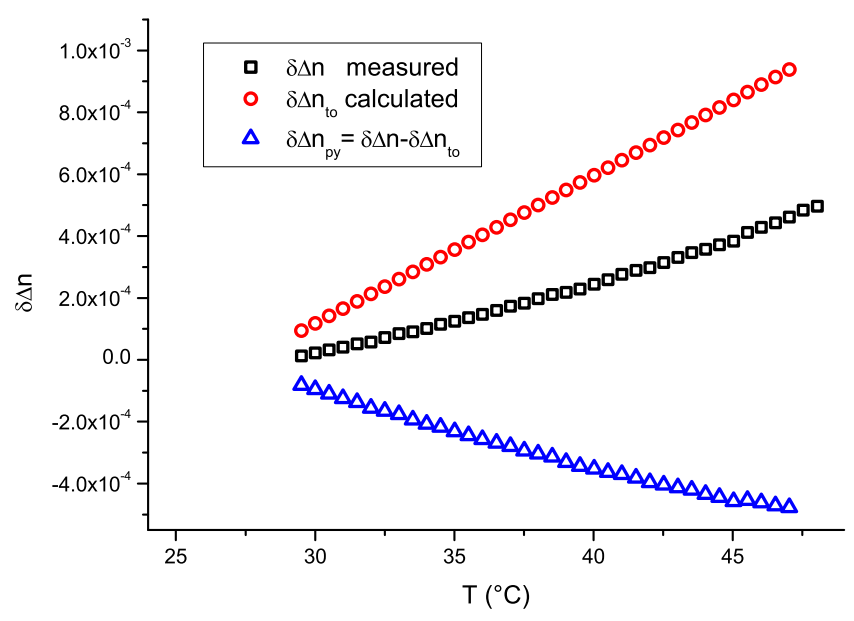

FIG. 3. (Color online) Typical birefringence variation in a stoichiometric crystal in open-circuit configuration. Total birefringence variation $\delta \Delta n$ (black squares), thermo-optic contribution $\delta \Delta n_{t o}$ (red circles), and pyroelectric contribution $\delta \Delta n_{p y}$ (blue triangles).

through Eq. (3) knowing the dielectric constants of $\mathrm{LiNbO}_{3}$, whose variation is also negligible in the considered temperature range. ${ }^{17}$

A typical behavior of $\Delta P_{s}$ is depicted in Fig. 4; finally the pyroelectric coefficient $p$ of the crystal is found from its derivative versus temperature. Specifically this derivative is calculated with a fitted curve to the experimental data of $\Delta P_{s}$ to avoid influence of noise measurement on evaluation of $p$. Note that, since the sample is mechanically unclamped during measurements, we obtain the total pyroelectric coefficient which is the sum of the primary and secondary pyroelectric coefficients.

For stoichiometric and congruent compositions six and four measurements are, respectively, performed in order to obtain an averaged values of the pyroelectric coefficients. We estimate an uncertainty of $12 \%$ on the measurements, due mainly to imperfections such as thermal control or setup alignment. These values of $p$ extrapolated for a temperature of $25{ }^{\circ} \mathrm{C}$ are presented in Table I and they are consistent with values measured with usual dielectric techniques, ${ }^{6-8,10}$ being the pyroelectric coefficients reported here in the high-

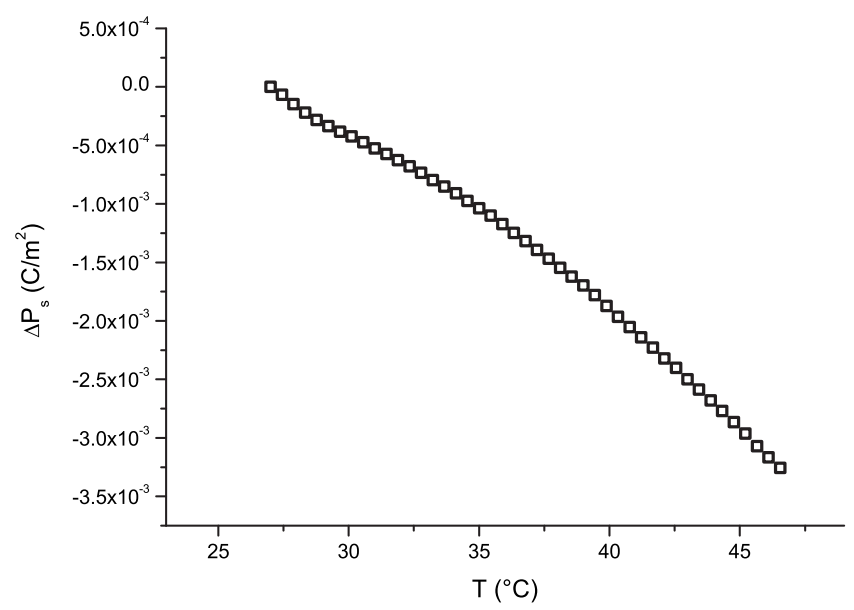

FIG. 4. Typical behavior of the spontaneous polarization variation $\Delta P_{s}$ in a congruent crystal.
TABLE I. Measured values of the unclamped pyroelectric coefficient $p$ at $25{ }^{\circ} \mathrm{C}$. The uncertainty is $12 \%$.

\begin{tabular}{lc} 
Sample & $p$ \\
\hline Congruent & $\left(\times 10^{-5} \mathrm{C} / \mathrm{m}^{2} \mathrm{~K}\right)$ \\
Stoichiometric & -10.39 \\
\hline \hline
\end{tabular}

value range among the ones found in the literature. Furthermore, the absolute value of $p$ for the congruent sample is found to be higher than the stoichiometric one, in accordance with the trend reported in the literature. ${ }^{8}$

Since we consider a rather small temperature interval $\left(20^{\circ} \mathrm{C}\right)$, in our conditions the pyroelectric coefficient temperature-correction $(\partial p / \partial T) \Delta T$ can be estimated of the order of $10 \%,{ }^{8,10}$ which is within our uncertainty. Therefore, we give only the temperature-independent term of $p$; for an estimation of the $\partial p / \partial T$ contribution a wider temperature interval have to be considered. However, this optical method leads to realistic values of the pyroelectric coefficients for $\mathrm{LiNbO}_{3}$ by using a temperature variation much smaller than those usually considered in the literature, which validates the proposed technique.

\section{CONCLUSIONS}

We have proposed a new all-optical technique to measure the pyroelectric coefficient of electro-optic crystals. It consists in measuring the mutual refractive indices variation induced by the pyroelectric field as a function of temperature change. As an example we use a Sénarmont experimental system to estimate the refractive birefringence change in $\mathrm{LiNbO}_{3}$. Inferred values of the pyroelectric coefficients $p$ of congruent and stoichiometric $\mathrm{LiNbO}_{3}$ samples are found to be very close to the ones measured with usual dielectric techniques, despite the fact that they are obtained by considering temperature intervals much smaller than those usually used. The proposed technique can be used to evaluate the pyroelectric properties of a ferroelectric crystal without need to place any electrodes. Moreover, since a localized beam is employed, this method has the potential to measure spatial variation in pyroelectricity in bulk crystals. The measurement is indeed localized to the light beam propagation path which allows a scanning of the beam to provide the pyroelectricity distribution, which is not accessible with usual dielectric techniques. This all-optical technique represents a powerful method to evaluate the pyroelectricity of electrooptical crystals complementary to dielectric techniques.

\section{ACKNOWLEDGMENTS}

This work was partially supported by FIRB "PHOCOS," Prot. RBFR08E7VA, and by Fondazione CARIPLO, Rif. 2007.5193.

${ }^{1}$ K. C. Kao, Dielectric Phenomena in Solids (Elsevier Academic Press, San Diego, London, 2004).

${ }^{2}$ L. Arizmendi, Phys. Status Solidi A 201, 253 (2004).

${ }^{3}$ S. M. Kostritskii, O. G. Sevostyanov, M. Aillerie, and P. Bourson, J. Appl. Phys. 104, 114104 (2008).

${ }^{4}$ J. Safioui, F. Devaux, and M. Chauvet, Opt. Express 17, 22209 (2009). 
${ }^{5}$ A. M. Glass, Phys. Rev. 172, 564 (1968).

${ }^{6}$ P. C. Barbosa, J. A. C. Pavia, F. Mendes, A. C. Hernandes, J. P. Andreeta, and A. S. B. Sombra, Phys. Status Solidi A 125, 723 (1991).

${ }^{7}$ J. S. Kim, J. B. Kim, B. C. Choi, C. S. Kim, J. H. Ro, and J. N. Kim, J. Korean Phys. Soc. 24, 343 (1991).

${ }^{8}$ T. Bartholomäus, K. Buse, C. Deuper, and E. Krätzig, Phys. Status Solidi A 142, K55 (1994).

${ }^{9}$ V. Daniel, Dielectric Relaxation (Academic Press, New York, 1967).

${ }^{10}$ S. V. Yevdokimov, R. I. Shostak, and A. V. Yatsenko, Phys. Solid State 49, 1957 (2007).

${ }^{11}$ M. Aillerie, M. D. Fontana, F. Abdi, C. Carabatos-Nedelec, N. Theo- fanous, and G. Alexakis, J. Appl. Phys. 65, 2406 (1989).

${ }^{12}$ M. Aillerie, F. Abdi, M. D. Fontana, N. Théofanous, and E. Abarkan, Rev. Sci. Instrum. 71, 1627 (2000).

${ }^{13}$ J. Zaccaro, J. P. Salvestrini, A. Ibanez, P. Ney, and M. D. Fontana, J. Opt. Soc. Am. B 17, 427 (2000).

${ }^{14}$ P. Minzioni, I. Cristiani, V. Degiorgio, and E. P. Kokanian, J. Appl. Phys. 101, 116105 (2007)

${ }^{15}$ T. R. Volk and M. Wöhlecke, Ferroelectr. Rev. 1, 195 (1998).

${ }^{16} \mathrm{P}$. Górski, R. Ledzion, K. Bondarczuk, and W. Kucharczyk, OptoElectron. Rev. 16, 46 (2008).

${ }^{17}$ R. T. Smith and F. S. Welsh, J. Appl. Phys. 42, 2219 (1971). 